diagnosis and deprivation were included in the model $(\mathrm{OR}=1.0390 ; 1.0133-1.0648, \mathrm{p}=0.003)$.

No significant associations were identified between social support and physical activity, diet, alcohol or smoking.

Conclusions Social support may be an important facilitator for attendance at appointments and adherence to CVD medications; however no association was found between social support and lifestyle behaviours. Harnessing positive social relationships in the delivery of interventions for reducing CVD risk in this population should be considered as a strategy for encouraging uptake.

\section{LB2 APPLYING MACHINE LEARNING TO POOLED QUALITATIVE STUDIES ON ACTIVE TRAVEL: A METHOD TO UNCOVER UNANTICIPATED PATTERNS TO INFORM BEHAVIOUR CHANGE?}

${ }^{1}$ E Haynes, ${ }^{1} \mathrm{R}$ Garside, ${ }^{2} \mathrm{~J}$ Green, ${ }^{3} \mathrm{MP}$ Kelly, ${ }^{4} \mathrm{~J}$ Thomas, ${ }^{1} \mathrm{C}$ Guell*. ${ }^{1}$ European Centre for Environment and Human Health, University of Exeter Medical School, Truro, UKi ${ }^{2}$ School of Population Health and Environmental Sciences, King's College London, London, UK; ${ }^{3}$ Cambridge Institute of Public Health, University of Cambridge, Cambridge, UK; ${ }^{4}$ Institute of Education, University College London, London, UK

\subsection{6/jech-2018-SSMabstracts.86}

Background Innovative approaches are required to better address physical inactivity. To move beyond individual approaches to behaviour change and develop more appropriate insights for the complex challenge of increasing population levels of activity, recent research has drawn on social practice theory. This theoretical approach describes the relational character of active living and related social practices. However, to date these investigations have been limited to small-scale qualitative research studies. To move beyond individual contexts and population groups and uncover conditions for 'practice change' across similar datasets, we explored a novel approach to qualitative data synthesis. Our aim was to pool several qualitative studies and apply machine learning to uncover patterns and interconnections in 'active travel' that have not emerged from the original qualitative data analyses.

Methods A pooled qualitative dataset of almost 250 transcripts was drawn from five studies conducted in different contexts in the UK, including Belfast, London, Glasgow, Cambridge and Cardiff. Machine learning approaches such as text mining have previously been applied to identify key recurring terms in large data sets. Recent software developments suggest the possibility of identifying 'concepts within context'. This unsupervised analysis of inter-relating concepts, which focuses on pattern recognition, is known as 'topic modelling analysis'. Text mining analysis software, Leximancer, was used to analyse the data and produce inter-topic distance maps to illustrate 'themes' and constituent 'concepts'.

Results In our analysis, we interrogated the insightfulness of this software to facilitate an inductive, data-driven process, and provide an analytical 'fresh lens' and the potential for identifying novel patterns and linkages that might not be identified by manual coding. For example, a new 'uncovered' theme was that women's accounts of 'cycling' were closely connected to 'people'. Exploring this in the original data, this related to their notions of who is a 'cyclist', what 'cyclists' look like, and aspects such as required fitness. In contrast, for men, 'cycling' did not connect to 'people' but to logistics, how to get to work and how long it takes. This researcher input and interpretative work was a necessary analytical next step to make meaning from software outputs.

Conclusion This study contributes new insights into the, to date, rare application of machine learning to qualitative social science research, and towards a social science approach to behaviour change. Developing new methods and conceptual understandings can inform future research and policy decisions about social environments for promoting social practices which increase physical activity.

Research funded by the Academy of Medical Sciences and the Wellcome Trust: Springboard - Health of the Public 2040 (HOP001\1051).

\section{LB3 BUILDING INTERNATIONAL CONSENSUS ON GUIDELINES FOR RESEARCHERS INTERACTING WITH THE FOOD INDUSTRY: SYSTEMATIC SCOPING REVIEW, DELPHI STUDY AND INTERNATIONAL WORKSHOP}

K Cullerton, J Adams, N Forouhi, O Francis, M White*. Centre for Diet and Activity Research, MRC Epidemiology Unit, University of Cambridge, Cambridge, UK

\subsection{6/jech-2018-SSMabstracts.87}

Rationale Most food in the UK comes from commercial food companies, which contribute to the environments that drive what we eat. Many food companies need to change to support healthier diets. Population health researchers need to understand how food companies shape food choices and identify levers for change. This may require that researchers interact with industry. However, there is no explicit consensus among researchers regarding what constitutes acceptable or effective interaction. This has led to confusion over potential conflicts of interest, which could obscure findings and undermine scientific integrity. We aimed to build international consensus on what constitutes appropriate interactions between population health researchers and the food industry.

Methods We undertook a systematic scoping review of published and grey literature, and a two-stage international Delphi study of population health researchers. Scopus and Pubmed were searched to May 2017 for articles in English referring to principles guiding interactions between population health researchers and the food industry. We also asked experts to nominate documents, conducted an advanced Google search and hand searched reference lists. We thematically analysed included articles to saturation to derive principles. A tworound, online Delphi survey was undertaken to determine and build consensus on these principles, using a multi-pronged, purposeful approach to recruitment of researchers internationally. Consensus was defined as $80 \%$ agreement on each statement. Content analysis of qualitative feedback informed the second Delphi round. Findings provided the starting point for a two-day international workshop, in which we discussed a framework and principles for guidance.

Findings Our systematic review identified 56 principles in five key areas: governance of funding, risk assessment, maintaining standards of governance, ensuring transparency and improving publication standards. 100 researchers completed the first round of the Delphi and 92 the second. After round two, there was consensus on 39 statements (68\%). Detailed comments by participants helped to modify principles. The international workshop identified the need for guidance as a 'thinking tool' rather than set of rules, offering support for researchers to assess risks and manage potential conflicts of 
interest throughout the research process. Effective guidance will need the support of researchers, funders and journals.

Discussion This research has built consensus on the need for guidance, and identified an optimal approach for assessing risk, prevention and management of conflicts of interest in interactions between population health researchers and the food industry. Further work is needed to finalise, pilot test and seek endorsement for evidence informed guidance.

\section{LB4 INDUSTRY REACTIONS TO THE UK SOFT DRINKS INDUSTRY LEVY: UNPACKING THE EVOLVING DISCOURSE FROM ANNOUNCEMENT TO IMPLEMENTATION}

TL Penney*, J Adams, M White. On behalf of the NIHR PHR SDIL Evaluation Team

\subsection{6/jech-2018-SSMabstracts.88}

Background Within the context of a global movement toward taxes on sugary drinks, the Soft Drinks Industry Levy (SDIL) is unique in its construction - a two-tiered levy that aims to encourage industry to reformulate soft drinks. Industry decisions regarding reformulation will directly influence the health impacts of the levy, however how these reactions are covered in the media will also shape a wider public discourse on sugar and health. This work will examine the evolution of industry reactions to the levy from announcement to implementation, via articles published in news media and trade press.

Methods We searched the Factiva database of UK news media and trade press. A search strategy was used to identify articles related to sugar or soft drinks and related to the levy covering March 16th 2016 to March 31st 2018. Articles were screened using predefined criteria. Analyses included: (a) description of included articles by industry actor and (b) a longitudinal, case-based, thematic analysis of each industry actor.

Results 526 articles were included covering the ongoing reactions by nine soft drinks industry actors (e.g. AG Barr, Britvic, Coca-Cola European Partners) during six policy development milestones and two national events. Early results demonstrate a discourse of disagreement with the aims of the SDIL immediately after its announcement with emergent themes including 'no evidence that sugar taxes reduce obesity', 'the poor will suffer' and 'this will destroy industry and kill jobs'. Reactions also included contradictory themes such as 'most products are not impacted' and 'we support government actions on obesity'. Throughout the consultation phase and during the Brexit vote and snap election further themes emerged including 'threats of legal action' that were not always consistent across industry actors but dominated until Royal Assent for the legislation. Throughout the parliamentary process the discourse shifted toward acceptance of the levy and undertaking efforts to adapt including 'diversification and innovative marketing efforts' and various 'cost management actions to offset the levy'. As the implementation of the levy approached, acceptance was reinforced by additional themes that sought to ensure perceived profitability with 'claims of strong sales and profits' and 'calls for investment in a sector with clear growth'.

Conclusion The shifting discourse suggests that industry actors are continually navigating issues of public, government and commercial interests, which results in conflicting narratives. Further work is needed to explore the discourses surrounding other related actors such as government, civil society and academics.

\section{\begin{tabular}{|l|l}
\hline LB5 & IS THE DURATION OF THE PRECEDING INTER-
\end{tabular} PREGNANCY INTERVAL ASSOCIATED WITH OFFSPRING'S SIZE AT BIRTH? - ANALYSIS OF A UK POPULATION-BASED COHORT}

${ }^{1} \mathrm{~N}$ Ziauddeen* ${ }^{1} \mathrm{PJ}$ Roderick, ${ }^{2} \mathrm{NS}$ Macklon, 1,3NA Alwan. ${ }^{1}$ Academic Unit of Primary Care and Population Sciences, Faculty of Medicine, University of Southampton, Southampton, UK; ${ }^{2}$ Department of Obstetrics and Gynaecology, University of Copenhagen, Zealand University Hospital, Roskilde, Denmark; ${ }^{3}$ NIHR Southampton Biomedical Research Centre, University of Southampton and University Hospital Southampton NHS Foundation Trust, Southampton, UK

\subsection{6/jech-2018-SSMabstracts.89}

Background Short and long intervals between pregnancies have been associated with increased risk of adverse birth outcomes including low birth weight and stillbirth. Birthweight is an indicator of the in-utero environment and a key early life risk factor for long-term health outcomes such as obesity and cardiovascular disease. The World Health Organization recommended in 2005 waiting at least 24 months after a live birth before getting pregnant again. There are no UK guidelines on birth spacing. We aimed to investigate the association between duration of the inter-pregnancy interval between successive live birth pregnancies and risk of having a small-for-gestational age (SGA) or large-for-gestational age (LGA) baby.

Methods A population-based cohort of prospectively collected routine healthcare data for antenatal care between January 2003 and September 2017 (total $\mathrm{n}=82098$ pregnancies) at University Hospital Southampton, Hampshire, UK was used. Records of women with their first two singleton live-birth pregnancies were analysed ( $\mathrm{n}=15922$ women). Inter-pregnancy interval was defined as timing between a live birth and the next conception. SGA was defined as $<10$ th percentile weight and LGA as >90th percentile weight for gestational age. Logistic regression was used to examine the association between risk of SGA or LGA and inter-pregnancy interval. The models were adjusted for maternal age, ethnicity, highest educational qualification, employment status, baseline maternal BMI, between pregnancy change in maternal BMI, smoking status at second pregnancy booking appointment and conception following infertility treatment. Sensitivity analyses was conducted adjusting for SGA or LGA in previous pregnancies. Results Twelve percent of first pregnancy and 7\% of second pregnancy births were SGA. Seven percent of first pregnancy and $13 \%$ of second pregnancy births were LGA. Three percent of women each had SGA and LGA babies in both pregnancies. Compared to an interval of 24-35 months, there was a lower risk of SGA birth in second pregnancy with an interval of 12-23 months (adjusted OR 0.82, 95\% CI 0.69 to $0.98, \mathrm{p}=0.03)$. The association remained after adjusting for previous outcome of SGA in sensitivity analysis. No association was observed between risk of SGA with intervals of $<12$ or $\geq 36$ months or LGA and inter-pregnancy interval. Conclusion An inter-pregnancy interval of 12-23 months was associated with lower risk of SGA, however the duration of the interval was not associated with LGA risk. In high-income countries with relatively healthy pregnant population, further research considering the potential advantages of shorter optimal interval between pregnancies than that recommended by WHO is needed. 